\title{
The chemical composition of extremely metal-poor and carbon-rich star HD 112869
}

\author{
L. Začs, I.Diebele and O.Alksnis
}

Institute of Atomic Physics and Spectroscopy, University of Latvia, Raiņa bulvāris 19, LV-1586 Rìga, Latvia email: zacs@latnet.lv

\begin{abstract}
The preliminary results of abundance analysis are presented for extremely metalpoor carbon star HD $112869=\mathrm{TT}$ CVn $=$ CGCS 3319. The radial velocity was found to be $-137.7 \mathrm{~km} \mathrm{~s}^{-1}$. Our LTE abundance analysis supports an extremely low metallicity for TT CVn, $[\mathrm{Fe} / \mathrm{H}]=-3.2$, and a significant overabundance of carbon and neutron-capture elements. The ${ }^{12} \mathrm{C} /{ }^{13} \mathrm{C}$ ratio in the atmosphere of HD 112869 is high.
\end{abstract}

Keywords. Stars: AGB and post-AGB, stars: abundances, stars: individual: HD 112869

\section{Observations and analysis}

High-resolution spectra for HD $112869(\mathrm{C} 4,5 \mathrm{CH})$ and the comparison stars U Hya (C6,3; Abia et al. 2002) and HD 25408 (C5,3 J; Kipper et al. 1996) were obtained with the coudé échelle spectrometer MAESTRO fed by the $2 \mathrm{~m}$ telescope at the Observatory on the Terskol Peak in Northern Caucasus equipped with a CCD detector with a resolving power of $\sim 45000$. A total exposure time of $7200 \mathrm{~s}$ was made for HD 112869 on 29 January 2004. The spectrum covered region from 3600 to $10200 \AA$ in 85 wavelength bands. An LTE abundance analysis was made using the code WIDTH9 and measured equivalent widths of selected clean lines. The atmospheric parameters for the first iteration are adopted from Kipper (1992): $3700 \mathrm{~K}, 0.4(\mathrm{cgs}), 4.0 \mathrm{~km} \mathrm{~s}^{-1}, \mathrm{C} / \mathrm{O}=1.07$. The atmospheric model was calculated by T.Kipper using the code SCMARCS 21.1.

\section{Conclusions}

The heliocentric radial velocity of HD 112869 was found to be $-137.7 \mathrm{~km} \mathrm{~s}^{-1}$, close to the previous estimations (see Kipper 1992). With $\mathrm{M}_{b o l}=-3.4$ the star seems to be a good candidate for intrinsic (AGB) halo carbon stars. Our abundance calculations support the extremely low metallicity for HD 112869, $[\mathrm{Fe} / \mathrm{H}]=-3.2$. CNO elements relative to iron are significantly enhanced, however, abundance calculations for similar cool stars (A.Jorissen, private communications) revealed remarkable dependance of calculations from the carbon abundance of used atmospheric model. The isotopic ${ }^{13} \mathrm{CN}$ lines are not clearly visible in the spectrum of HD 112869 , therefore, a high ${ }^{12} \mathrm{C} /{ }^{13} \mathrm{C}$ ratio is confirmed. The neutron-capture elements relative to iron are enhanced by factors of more than 10 .

\section{References}

Abia, C., Domínguez, I., Gallino, R., Busso, M., Masera, S., Straniero, O., de Laverny, P., Plez, B., \& Isern, J. 2002, ApJ 579, 817

Kipper, T. 1992, BaltA 1, 181

Kipper, T., Jørgensen, U.G., Klochkova, V.G., \& Panchuk, V.E. 1996, A\&3A 306, 489 

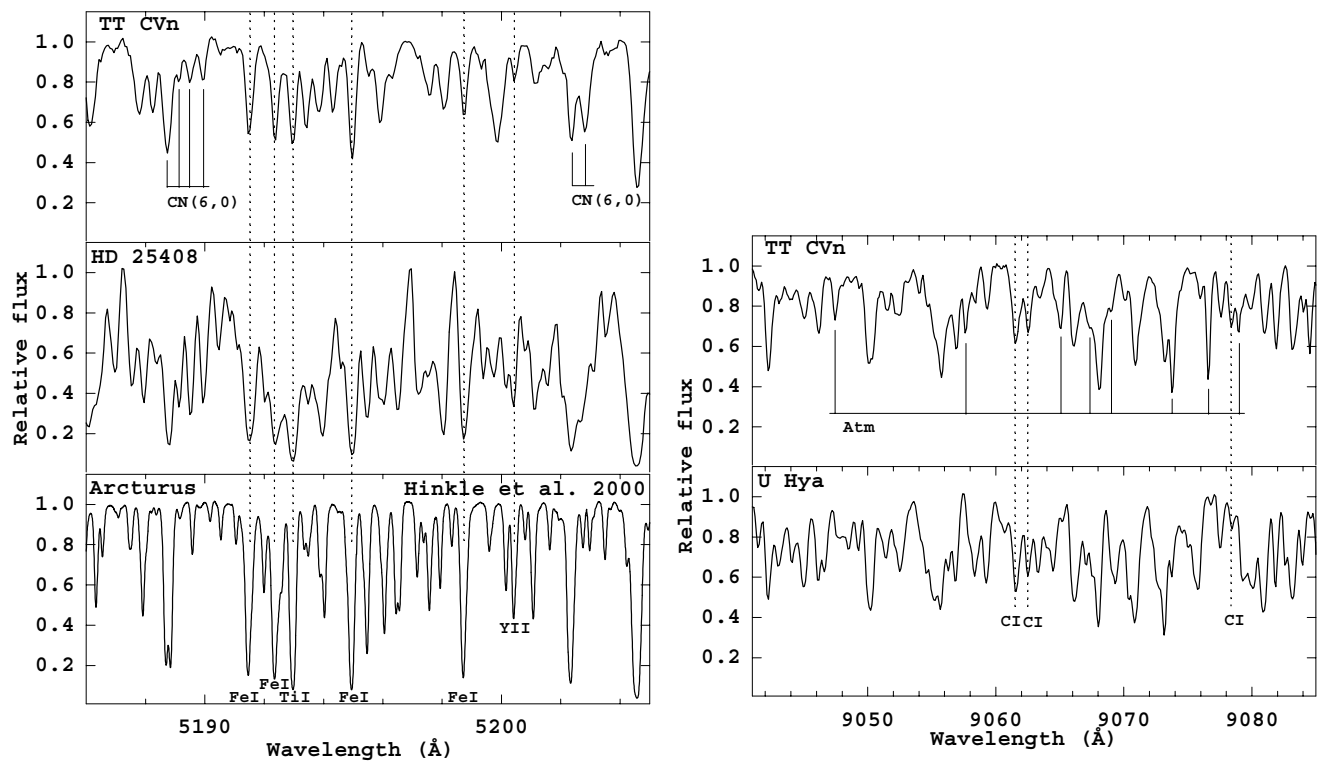

Figure 1. The observed spectra of HD $112869(\mathrm{C} 4,5 \mathrm{CH})$, along with those for the comparison stars HD 25408 (C5,3 J) and Arcturus, in two selected wavelength regions. Although HD 112869 is an extremely metal-poor, $[\mathrm{Fe} / \mathrm{H}]=-3.2$ and $[\mathrm{Ti} / \mathrm{H}]=-3.5$, a medium-high enhancement of the light $\mathrm{s}-$ process element $\mathrm{Y}$ was found, $[\mathrm{Y} / \mathrm{Fe}]=+1.3$. The preliminary calculations revealed a significant carbon overabundance relative to iron.
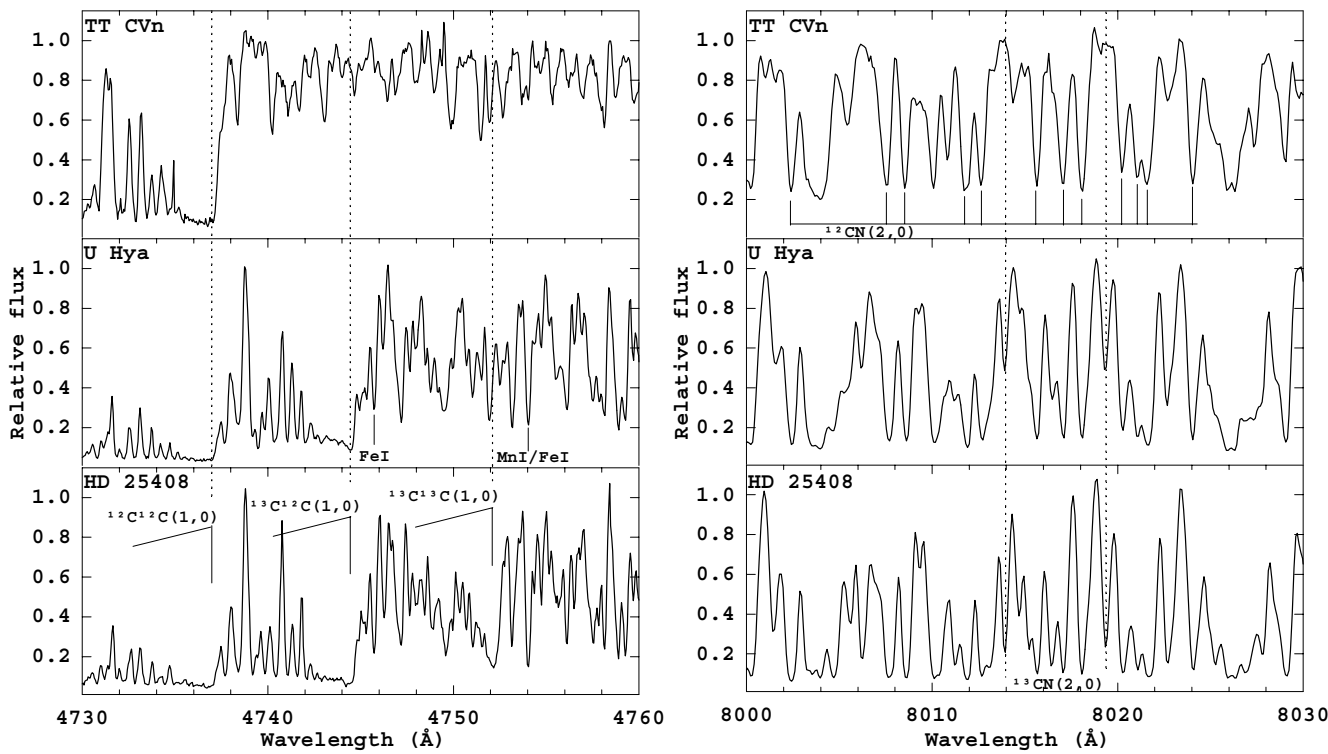

Figure 2. The spectrum of HD $112869(\mathrm{C} 4,5 \mathrm{CH})$, along with those for the comparison stars U Hya $(\mathrm{C} 6,3)$ and HD $25408(\mathrm{C} 5,3 \mathrm{~J})$, in the region of $C_{2}$ Swan system $(1,0)$ bandheads at 4740 $\AA$ (left) and around selected $\mathrm{CN}(2,0)$ lines near $8020 \AA$ (right). The isotopic features due to ${ }^{13} \mathrm{C}$ indicated by dotted lines in the spectrum of HD 112869 are not visible. Thus a high ${ }^{12} \mathrm{C} /{ }^{13} \mathrm{C}$ ratio is confirmed in comparison with $\mathrm{U}$ Hya $\left({ }^{12} \mathrm{C} /{ }^{13} \mathrm{C}=35\right)$ and $\operatorname{HD} 25408\left({ }^{12} \mathrm{C} /{ }^{13} \mathrm{C}=5\right)$. 


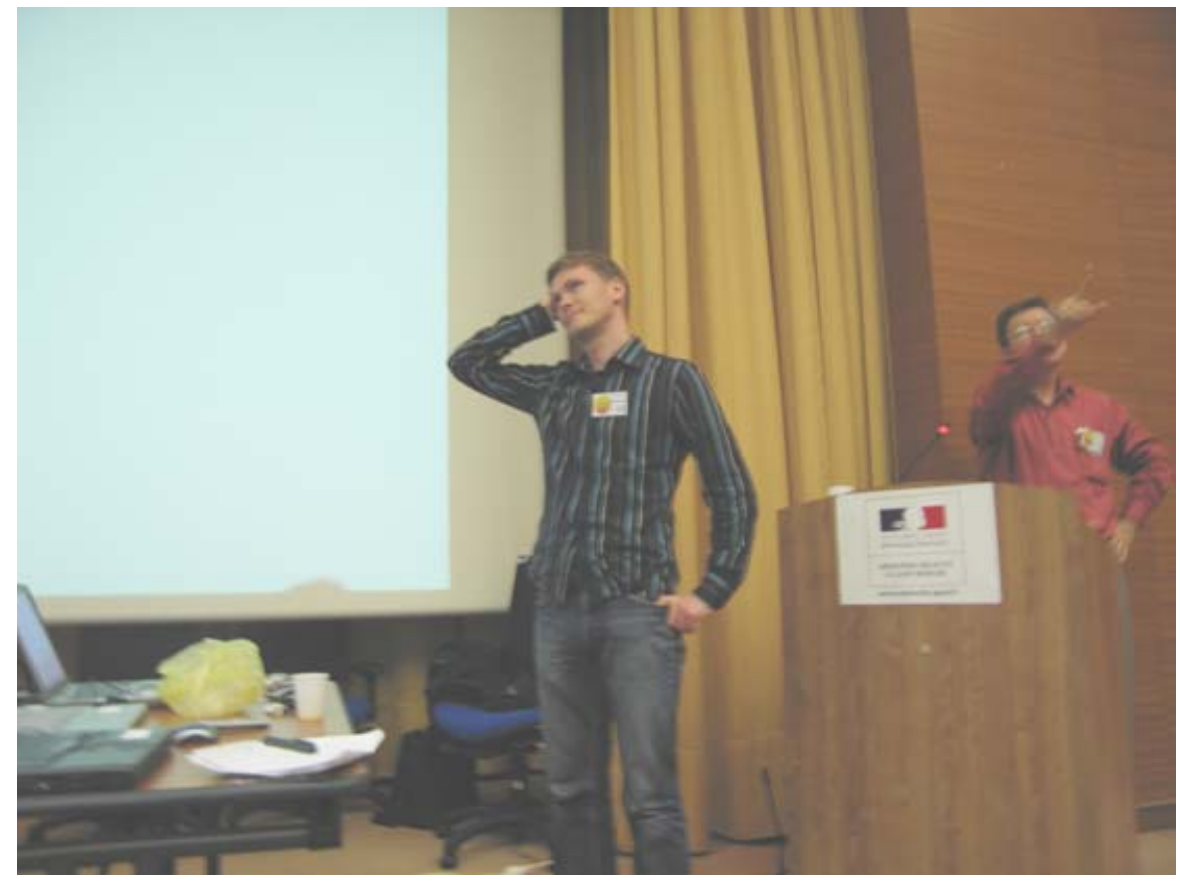

Torgny Karlsson presenting inhomogeneous chemical evolution models.

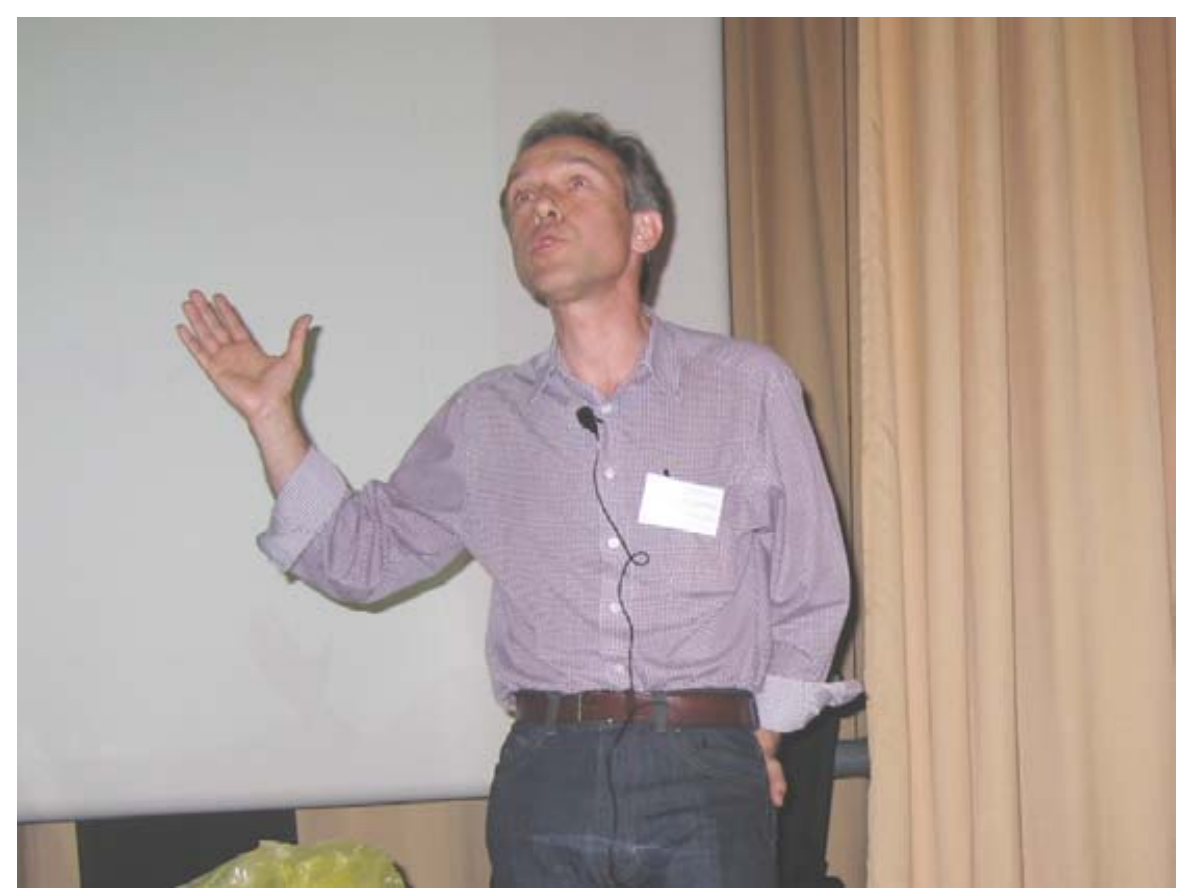

Nikos Prantzos answering questions. 


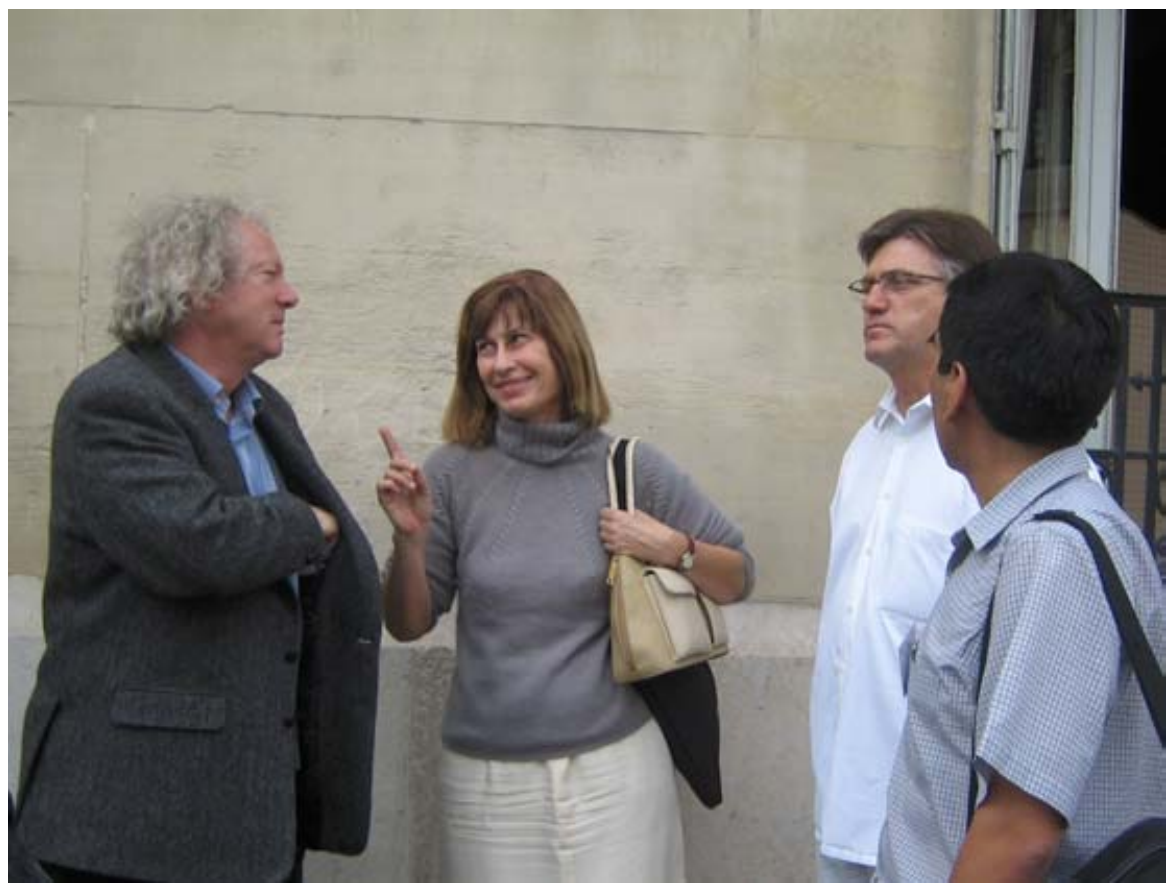

Waiting for the bus to the conference dinner, Mike Edmunds and Beatriz Barbuy discussing under the gaze of Verne Smith.

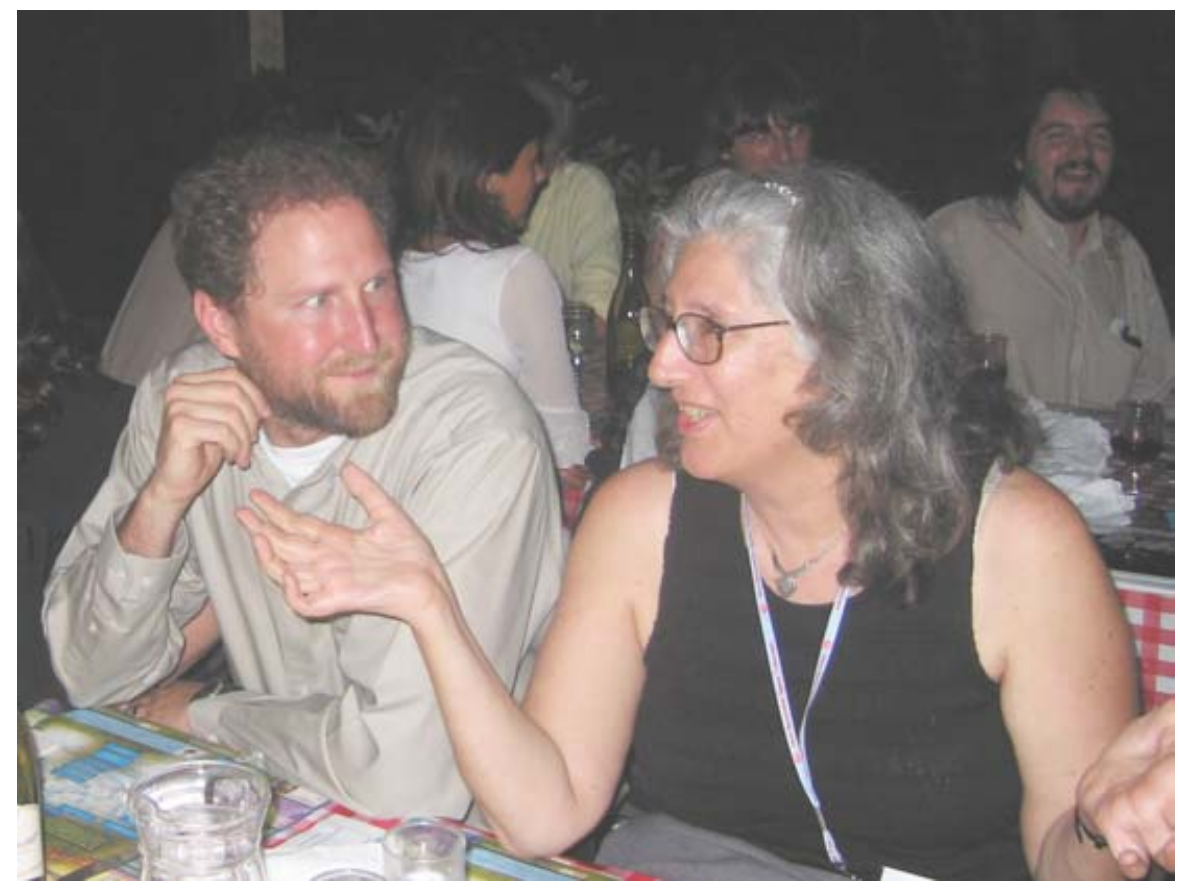

Matthew Shetrone listening to Judy Cohen at the conference dinner. 